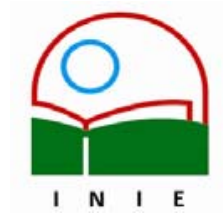

Universidad de Costa Rica

Facultad de Educación

Instituto de Investigación en Educación

ACTUALIDADES INVESTIGATIVAS EN EDUCACION

\title{
APORTE DE LA NUEVA MUSEOLOGÍA ESPAÑOLA AL MUSEO DE LOS NIÑOS DE COSTA RICA ${ }^{1}$
}

Keilyn Rodríguez Sánchez*

\begin{abstract}
Resumen: El artículo que se ofrece consiste en una comparación etnográfica sobre propuestas educativas no formales, entre el Museo de los Niños de Costa Rica y seis museos o centros de ciencia españoles ubicados en lo que se conoce como la nueva museología. Se detallan los aspectos de la nueva museología española que pueden enriquecer la práctica del museo costarricense.

Palabras claves: MUSEOLOGÍA EDUCACIÓN NO FORMAL MUSEOS INTERACTIVOS Y MUSEOS VIRTUALES/ COMPARACIÓN DE MUSEOS/

Abstract: The following article consists in an ethnographic comparison about non-formal educational proposals. This comparison is carried out between Museo de los Niños and other six museums or Science Centers located in Spain which are known as New Museology. Certain aspects in which the New Spanish Museology enriches the practice in Costa Rican museum are detailed.
\end{abstract}

Key words: MUSEOLOGYI NON-FORMAL EDUCATION/ INTERACTIVE MUSEUMS AND VIRTUAL MUSEUMS/ MUSEUM COMPARISON/

\section{Introducción}

El presente artículo ofrece una comparación de seis museos interactivos y centros de ciencia españoles, con el Museo de los Niños de Costa Rica, específicamente en el ámbito de la propuesta educativa, en relación con todas aquellas acciones planificadas y orientadas por objetivos y metas para contribuir con la educación de los visitantes del museo.

Los objetivos del estudio son los siguientes:

a. Comparar el Museo de los Niños de Costa Rica con seis museos interactivos o centros de ciencia españoles, que cuenten con su respectivo museo virtual, según su propuesta educativa no formal.

\footnotetext{
* Candidata a Doctora en Educación en la Universidad de Costa Rica, Licenciada en Antropología Social y en Ciencias de la Educación con énfasis en Administración de Programas de Educación no Formal, de la misma Universidad. Funcionaria del Museo de los Niños de Costa Rica.
}

Correo electrónico: joyke96@hotmail.com

Artículo recibido: 22 de julio, 2005

Aprobado: 31 de octubre, 2005

\footnotetext{
${ }^{1}$ Este estudio fue financiado con una beca de formación en la Universidad de Salamanca (España) otorgada por la Unión Europea mediante el Programa Alban con el no. E04D043003CR, y con la tutoría del Dr. Joaquín García Carrasco.
} 
b. Identificar actividades, proyectos y elementos museográficos españoles, que puedan realizarse o enriquecer la propuesta educativa del Museo de los Niños de Costa Rica.

Con este fin, se presentan los conceptos teóricos centrales del estudio y luego los antecedentes. Posteriormente, se expone el método de trabajo. Y, en los últimos puntos, se exponen los resultados de la comparación y propuesta para el Museo de los Niños de Costa Rica.

\section{Definiciones teórico conceptuales: los museos interactivos y los centros de ciencia}

Si bien existe diferencia entre la definición de "museo interactivo" y "centro de ciencia", para fines de este estudio se incluyen ambos tipos de instituciones para ser comparadas con el Museo de los Niños de Costa Rica, desde su producción educativa. La razón de esta inclusión radica en que el Museo de los Niños ha sido creado, como un híbrido de los dos primeros. Por lo tanto, dados los objetivos de comparación de este estudio, no interesa ni es posible excluir uno u otro.

Sin embargo, es necesario diferenciar, conceptualmente, a los museos interactivos de los centros de ciencia. Debido a lo anterior, podemos definir los museos interactivos, como instituciones educativas que tienen como fin exponer mediante, sus exhibiciones, dispositivos que puedan ser accionados o que permitan una interacción con la mayor cantidad de sentidos y habilidades cognitivas posibles, por parte de los educandos.

Los centros de ciencia son más especializados temáticamente, pues su objetivo es demostrar y dar a conocer el acervo científico (método, teorías y leyes) y técnico de occidente (Sabbatini, 2003, pp. 1-2).

Tanto los centros de ciencia como los museos interactivos comprenden la antesala de los museos virtuales ${ }^{2}$. Estos centros se manifiestan como una variedad de los otros o un complemento y no como su competencia.

Interesa detenerse en los museos virtuales, ya que ofrecen una fuente de información del presente estudio. Según Sabbatini (2003) los museos virtuales suelen presentar los siguientes componentes: 
1. Folleto virtual: Incluye información propia del museo físico con fines divulgativos.

2. Colecciones, exhibiciones y experimentos en línea: Las colecciones en línea suponen la posibilidad de búsqueda por medio de bases de datos y su acceso mediante la navegación desde la colección general. Esta presentación de los objetos facilita el acceso a gran cantidad de información que enriquece su vinculación con el contexto y permite al educando, explorar según la profundidad de sus intereses y sin tener que trasladarse al museo físico.

3. Visitas virtuales y ambientes de realidad virtual: Esta modalidad puede realizarse mediante un conjunto de páginas Web dispuestas, lógicamente para visitar algún aspecto del museo físico. Otra forma de representación es por medio de ambientes en tres dimensiones navegables, que ofrecen una sensación mínima de presencialidad. Y la última, es mediante un ambiente de simulación en tres dimensiones el cual facilita una libertad de interacción con representaciones del museo físico y con otros visitantes virtuales.

4. Materiales y actividades educativos: Es posible encontrar una oferta de actividades didácticas como recursos educativos para los docentes en su trabajo en el aula, así como experiencias educativas en línea para diversos visitantes y sobre diversos temas. En este sentido, suele ser conveniente explorar temas del museo, mediante complementos educativos virtuales (experimentos, simulaciones, demostraciones, entre otros).

Es importante destacar que tanto los museos interactivos, los centros de ciencia y los museos virtuales, conforman lo que se llama la nueva museología ${ }^{3}$, pues son instituciones que han cambiado, respecto del museo tradicional. Este cambio se da en relación al público al cual se dirigen, al tipo de colección, algunas veces, a la forma de exhibición, a la apertura en los temas que se tratan y a la dinámica que se espera que se presente entre los visitantes y la colección.

A continuación, se ofrece un cuadro resumen de estos aspectos, con base en la propuesta de Rodríguez (2000, pp. 6-11):

${ }^{2}$ Según Sabbatini (2003) los museos virtuales son una colección de objetos digitales organizados lógicamente, según el tema de interés, con métodos de interacción informatizados y con objetivos similares a los de cualquier museo, pero con acceso mundial mediante la Internet.

${ }^{3}$ En el ámbito mundial, se reconoce que los museos interactivos, los centros de ciencias, los museos comunitarios y los eco-museos son expresiones de la nueva museología, los cuales surgen como formas diferentes al quehacer museológico tradicional (Ariel, 2004) 


\title{
Comparación entre el museo tradicional y el museo interactivo
}

\author{
o centro de ciencia
}

\begin{tabular}{|c|c|c|}
\hline $\begin{array}{c}\text { Característica de } \\
\text { cambio }\end{array}$ & Museo tradicional & $\begin{array}{l}\text { Museo interactivo y centro de } \\
\text { ciencia (nueva museología) }\end{array}$ \\
\hline $\begin{array}{l}\text { Público al cual se } \\
\text { dirige la exhibición }\end{array}$ & $\begin{array}{l}\text { Público con educación } \\
\text { especializada para interpretar los } \\
\text { objetos museales. }\end{array}$ & $\begin{array}{l}\text { Público común. Se diseña para el } \\
\text { pueblo. }\end{array}$ \\
\hline Tipo de colección & $\begin{array}{lcrr}\text { Piezas únicas } & \text { y } & \text { piezas } \\
\text { originales de } & \text { gran } & \text { valor } \\
\text { patrimonial, por lo } & \text { general } \\
\text { aisladas de contexto. } & \end{array}$ & $\begin{array}{l}\text { Son dispositivos de diversa } \\
\text { índole, con las características de } \\
\text { un juguete. Suelen ser réplicas. }\end{array}$ \\
\hline $\begin{array}{l}\text { Temas de } \\
\text { exhibición }\end{array}$ & Lo "culto" o de interés de la elite. & $\begin{array}{l}\text { Cualquier producción humana en } \\
\text { sociedad, es un tema de } \\
\text { exhibición. Interesa dar a } \\
\text { conocer las culturas populares y } \\
\text { también aspectos científicos de } \\
\text { la cultura universal, } \\
\text { específicamente en los centros } \\
\text { de ciencia. }\end{array}$ \\
\hline $\begin{array}{l}\text { Forma de } \\
\text { exhibición }\end{array}$ & $\begin{array}{l}\text { Se enfoca el objeto, usualmente } \\
\text { sin texto o el mínimo posible. Se } \\
\text { presenta una gran cantidad de } \\
\text { objetos y se pueden repetir. Es } \\
\text { necesario conocer códigos } \\
\text { especializados para interpretar la } \\
\text { colección. }\end{array}$ & \begin{tabular}{|lr} 
Se enfoca el contexto de los \\
objetos por su uso o por el tema \\
al que se refiere. & No es \\
necesario conocer códigos \\
especializados & para \\
interpretarlos. & \\
\end{tabular} \\
\hline $\begin{array}{l}\text { Relación visitantes- } \\
\text { colección }\end{array}$ & $\begin{array}{l}\text { La tendencia es admirar y } \\
\text { observa las colecciones, con una } \\
\text { actitud de respeto y silencio. } \\
\text { Interesa que el visitante lea la } \\
\text { exhibición desde sus } \\
\text { conocimientos especializados, } \\
\text { pues solo existe una forma } \\
\text { correcta de interpretación. }\end{array}$ & $\begin{array}{l}\text { La tendencia es utilizar la mayor } \\
\text { cantidad de habilidades físicas y } \\
\text { cognitivas, con actitud de } \\
\text { sorpresa y juego. } \\
\text { Interesa que el visitante se } \\
\text { planteé hipótesis sobre lo que } \\
\text { observa; se planteé preguntas } \\
\text { que no, necesariamente se } \\
\text { resolverán en la visita y que } \\
\text { construya su propio criterio sobre } \\
\text { el tema de la exhibición. }\end{array}$ \\
\hline
\end{tabular}

Así, tal y como afirma Pastor (2004, pp. 53-55), en la museología tradicional se asume que el conocimiento existe objetivamente y que puede ser transmitido al aprendiz en un proceso pasivo, asimilativo, sumativo, sistemático y linealmente estructurado.

Por otro lado, en la nueva museología, se procura partir de las necesidades educativas de los visitantes; facilitar pautas para interpretar los objetos; ofrecer programas educativos con diversas modalidades que permitan la forma deseada por los educandos para conseguir 
información, favorecer el establecimiento de conexiones entre el contenido y las preconcepciones; y animar al visitante a elaborar sus propias conclusiones sobre la problemática tratada en las exposiciones.

\section{La educación en los museos}

El museo, como creación occidental, ha sido concebido como una institución educativa desde sus orígenes, pese a que estaba destinada a una elite y a que no se ofrecía información o facilitaba el acceso, a quienes no conocieran el tema de lo expuesto.

Con la nueva museología, como se ha indicado, lo que interesa es transformar el enfoque tradicional y permitir el acceso a más grupos sociales, mediante un replanteamiento de los temas por tratar y de la forma didáctica de hacerlo. Tal y como se pudo observar en el cuadro presentado en la sección anterior, este cambio permite mayor cantidad y variedad de interacciones entre los educandos y la exhibición, esperando como resultado, mayores situaciones de aprendizaje y un acercamiento de mas grupos sociales a la institución museo.

Así, estas nuevas exposiciones llamadas interactivas que están compuestas por objetos museográficos o juguetes, son diseñadas para tocar, jugar, oler, saltar, clasificar, ver y comparar, armar, participar, relacionar, plantearse hipótesis, experimentar, cuestionar, entre otras actividades físicas y cognitivas que faciliten el aprendizaje, según los objetivos propuestos.

Falk y Dierking (2000) en "Learning from Museums" destacan dos características pedagógicas centrales que deben mantener los museos interactivos. La primera hace referencia al poder de escogencia departe del educando, en tanto tenga la libertad de manejarse en el museo, siguiendo su propio interés. Afirman que ese "interés" funciona como el filtro cerebral del foco de atención y, por lo tanto, facilita la persistencia en una tarea, la continua curiosidad por explorar aquello que se desea conocer y ese es un comportamiento que facilita el aprendizaje.

La segunda característica que exponen los autores, consiste en el hecho de que esas interacciones orientadas, pedagógicamente, por la museografía, se deben provocar en un contexto de emociones agradables, tales como: la sorpresa ante lo desconocido, el humor, el juego y en un ambiente diseñado para ser atractivo y agradable.

Una de las manifestaciones educativas que se evidencia y fortalece con la creación de los museos interactivos y centros de ciencia, es la implementación de actividades propias de la educación no formal o pedagogía social de complemento (que además le dan vitalidad al lugar) al cumplimiento de los objetivos de la institución. En este contexto, resulta 
prácticamente imposible en la actualidad encontrarse con un museo "interactivo" (como la descrita en el párrafo anterior) que no cuente con un departamento o unidad dedicada a efectuar actividades educativas, relacionadas con la exhibición permanente.

\section{Contexto del estudio}

Debido al particular interés que se expone en este estudio, no se ha registrado un antecedente de estudios similares. Sin embargo, existe una investigación que sirve de punto de partida y referente de comparación. Así, lo que respecta al análisis de museos virtuales no se contempla en la guía de observación para la comparación de museos, sino que se retoma del trabajo de Sabbatini (2004).

Sabbatini expone una investigación experimental para determinar la situación de los museos virtuales en Ibero América, España y Portugal, respecto a la oferta digital. Así, lo que se trabaja es una comparación de páginas Web de museos virtuales, para determinar los alcances del uso de este recurso.

En lo referido al caso de España, Portugal (Península Ibérica) y Latinoamérica se ofrece un cuadro resumen que muestra características de las páginas Web que permiten comparar a esos países, según el peso porcentual:

\section{Comparación de museos virtuales de España y Portugal con Latinoamérica}

\begin{tabular}{|l|c|c|}
\hline \multicolumn{1}{|c|}{ Característica de comparación } & España y Portugal & Latinoamérica \\
\hline Dominio propio. & $63.2 \%$ & $78.2 \%$ \\
\hline Misión del sitio Web & $100 \%$ & $5.9 \%$ \\
\hline Idiomas & $1: 38.7 \%$ & $1: 94.1 \%$ \\
& $2: 33.3 \%$ & $2: 5.9 \%$ \\
& 3 ó 4: $27.8 \%$ & \\
\hline Actualización y mantenimiento del sitio Web & $5.6 \%$ & $17.7 \%$ \\
\hline Dirección general de contacto & $72.2 \%$ & $74.5 \%$ \\
\hline Plano del sitio Web o índice & $16.7 \%$ & $5.88 \%$ \\
\hline Mecanismo de búsqueda & $11.1 \%$ & $17.7 \%$ \\
\hline Noticias o actualidad de la vida del museo & $77.8 \%$ & $82.3 \%$ \\
\hline Anuncio de requisitos técnicos para acceder & $11.1 \%$ & $17.7 \%$ \\
todos los espacios en la Web & $11.1 \%$ & - \\
\hline Site tour: resumen de contenidos del Web & $16.8 \%$ & $5.9 \%$ \\
\hline Información para prensa & Volumen 5, Número 2, Año 2005, ISSN 1409-4703 6 \\
\hline
\end{tabular}




\begin{tabular}{|c|c|c|}
\hline Indicación a un amigo & $100 \%$ & $5.9 \%$ \\
\hline Información sobre investigación & $16.7 \%$ & $35.3 \%$ \\
\hline Visita virtual & $33.3 \%$ & $17.7 \%$ \\
\hline Información sobre exposiciones & $100 \%$ & $100 \%$ \\
\hline $\begin{array}{l}\text { Exposiciones en línea de exposiciones } \\
\text { físicas }\end{array}$ & $16.7 \%$ & $17.6 \%$ \\
\hline $\begin{array}{l}\text { Exposiciones en línea sin relación con } \\
\text { exposiciones físicas }\end{array}$ & $11.1 \%$ & $17.6 \%$ \\
\hline Colecciones en línea & $5.6 \%$ & $5.9 \%$ \\
\hline Actualidad y noticias científicas & $22.2 \%$ & $11.8 \%$ \\
\hline $\begin{array}{l}\text { Vídeo como complemento (ninguno con } \\
\text { audio) }\end{array}$ & $5.6 \%$ & - \\
\hline Entornos de realidad virtual 3D & $5.6 \%$ & - \\
\hline Páginas para niños & $5.6 \%$ & $5.9 \%$ \\
\hline Materiales para profesores & $11.1 \%$ & $23.5 \%$ \\
\hline Materiales para estudiantes & $16.7 \%$ & $23.5 \%$ \\
\hline Experimentos virtuales & $11.1 \%$ & $17.6 \%$ \\
\hline Juegos y pruebas & $22.2 \%$ & $35.3 \%$ \\
\hline $\begin{array}{l}\text { Agentes inteligentes: mediante técnicas de } \\
\text { inteligencia artificial y personajes } \\
\text { antropomórficos para acceder a la } \\
\text { información deseada }\end{array}$ & - & $5.9 \%$ \\
\hline Sala de chateo y foro de discusión & $2.8 \%$ & $8.8 \%$ \\
\hline
\end{tabular}

Es importante aclarar que si bien el autor ofrece muchos más datos, para efectos del presente estudio, se considera que estos son los necesarios, puesto que presentan una mayor relación con lo educativo. Sabbatini (2004, p. 436) destaca con sus datos que en el caso de Ibero América los recursos tecnológicos presentes en los museos virtuales, son mucho más escasos que en los museos de la península lbérica.

Por lo tanto, es posible notar como el medio virtual es un espacio sumamente desaprovechado, por parte de los museos iberoamericanos no tradicionales. También se puede observar, cómo el desarrollo educativo, en ambos casos, es similar en lo que respecta al avance en museos virtuales. 
Sin embargo, a diferencia de Latinoamérica, se puede notar como la península lbérica tiene gran ventaja respecto a la variedad de lenguas, en la cuales sus páginas se encuentran disponibles y, además, le ofrece al navegante su misión y su estructura del sitio de manera más clara. Lo mismo sucede con las visitas virtuales y el acceso a la información actualizada sobre lo que sucede en el museo físico.

Ahora bien, Latinoamérica por su parte, se ha centrado más que la península Ibérica, en el ofrecimiento de actividades educativas para estudiantes, profesores, así como juegos, pruebas y experimentos. En este sentido, llama la atención que es solo en esta región, donde se trabaja con los llamados agentes inteligentes.

Es importante señalar que estas diferencias no evidencian el aprovechamiento educativo de este recurso por parte de las poblaciones respectivas. De hecho Sabbatini (2004, p. 43) se refiere al poco provecho del museo virtual como recurso educativo y divulgativo. Este autor muestra como existe un común perfil de retraso en Ibero América, en comparación con algunos casos de páginas Web estadounidenses y del resto de Europa. Sin embargo, la pregunta inmediata sería: ¿qué aprovechamiento real tendría este recurso en Latinoamérica y la Península Ibérica? Lo cual ofrece la oportunidad de proponer un estudio de factibilidad educativa como punto de partida.

Este estudio que presenta Sabbatini (2004) sobre museos virtuales refleja claramente la realidad del museo virtual del Museo de los Niños.

Uno de los aspectos centrales que destaca Sabbatini (2004) y, con el cual la autora está totalmente de acuerdo, es la ausencia de información en los museos virtuales, en ambas regiones, sobre museología científica, revistas científicas electrónicas y proyectos colaborativos que profundicen en el estudio y mejoramiento del quehacer museográfico y de actividades educativas complementarias.

\section{Método de trabajo}

Tal y como se indicó en la introducción, interesa efectuar un estudio comparativo entre el Museo de los Niños de Costa Rica, con seis museos interactivos o centros de ciencia españoles, que cuenten con su respectivo museo virtual, respecto a su propuesta educativa no formal, así como identificar actividades, proyectos y elementos museográficos españoles que puedan realizarse o enriquecer los existentes en la otra institución.

Por consiguiente, se realizará un estudio de casos comparativo, mediante la descripción cualitativa, el cual se ha organizado, según los siguientes pasos: 
1. Selección de los museos interactivos y centros de ciencia españoles, tomando en consideración los siguientes aspectos:

a. Instituciones que cuenten con un museo virtual que facilite parte del acceso a la información que se quiere revisar (Sabbatini, 2004).

b. Instituciones reconocidas en el ámbito nacional e internacional, como parte de la nueva museología.

c. Recomendaciones de expertos en la temática.

En este sentido, los museos y los centros de ciencia españoles que se seleccionaron son los siguientes:

- Museo de Ciencias Naturales de Barcelona (MCNB).

- Museo Nacional de Ciencias Naturales en Madrid (MCNM).

- Museo de la Ciencia en Madrid, Cosmocaixa (CM).

- Museo de la Ciencia en Barcelona, Cosmocaixa (CB).

- Parque de las Ciencias en Granada (PCG).

- Ciudad de las Artes y de las Ciencias en Valencia (CACV).

2. Diseño de una guía de observación etnográfica, con base en las propuestas educativas del Museo de los Niños de Costa Rica. Ver anexo I. El objetivo de la guía de investigación es efectuar una descripción detallada y organizada sobre: los objetivos y políticas institucionales, la estructura organizacional, la museografía, las actividades educativas, las formas de financiamiento y la investigación y evaluación de programas educativos. Se parte de lo que se conoce para luego comparar. Es importante señalar que no se ha registrado durante varios años de búsqueda bibliográfica, sobre el tema de museos, ningún tipo de clasificación similar que permita servir como una guía a la propuesta que se ofrece.

La observación etnográfica, según Spradley (1980), supone un proceso de comprensión por parte del investigador de lo que desea conocer, mediante la observación participante, las conversaciones con los actores involucrados en la escena cultural, en este caso del museo o centro de ciencia, y la revisión de cualquier otra fuente de información que sea útil. Para este autor, el conocimiento cultural debe ser sistematizado en matrices o estructuras analíticas que permitan la comunicación sistemática de lo que se estudia. Por esta razón, se utiliza una guía de observación y un paradigma para comparar. 
La comparación se efectúa para identificar diferencias y semejanzas por categoría y para ver si existe alguna área no implementada en el Museo de los Niños de Costa Rica y que sea de interés para esta institución, con base en su realidad particular. Esta guía se validó de la siguiente manera:

a. Con al menos una (jefatura) y máximo dos colaboradores (jefatura y colaborador) de cada una de las unidades educativas del Museo de los Niños de Costa Rica, según su especialización. La sección de museografía revisó los contenidos referidos únicamente a la categoría de "museografía" y así sucesivamente. Se consultaron los siguientes aspectos:

- ¿Cree que esta descripción está bien planteada según su experiencia de trabajo?

- Si cree que esta descripción no abarca todos los aspectos ¿cuáles considera que quedan por fuera?

- Comentarios

Con esta validación, se enriqueció la descripción de las categorías que se ofrecen en el anexo I, específicamente la de "museografía", ya que por la diversidad de componentes resultaba de mayor dificultad. Sin embargo las otras categorías también fueron revisadas y no se sugirieron cambios.

b. Con dos investigadores para asegurar la calidad del diseño de la guía y la armonía con la propuesta.

3. Revisión del sitio Web de cada una de las instituciones. Con base en la información disponible, se diseña una guía para las entrevistas necesarias en cada institución. Se visitaron lugares que contaban con sitios Web, ya que esto resuelve en parte la limitación de tiempo de estadía en cada institución.

4. Visita a cada institución como observadora participante de la exposición propiamente y si era posible con alguna persona encargada de aspectos educativos. Se consultó toda la información impresa disponible en cada lugar, y por último, una entrevista con al menos un miembro de cada una de las dependencias vinculadas de manera directa con tareas educativas.

5. Análisis de resultados en el siguiente orden:

a. Breve descripción de cada una de las instituciones visitadas. Utilizando como eje aspectos generales propios de cada una que permitan una ubicación al lector. 
b. Con base en la guía de observación, se elaboró un paradigma comparativo, el cual se diseñó para indicar la presencia, ausencia o no pertinencia de rasgos. (ver anexo II), se efectuó una descripción únicamente de los rasgos ausentes para el caso del Museo de los Niños de Costa Rica. De esta manera, se plasmó la comparación.

c. De acuerdo con lo anterior se irá ofreciendo un análisis crítico del posible aporte de la experiencia de la nueva museología española al Museo de los Niños de Costa Rica.

\section{Los museos seleccionados}

En este apartado se ofrece un breve resumen de las características generales de los museos seleccionados para el estudio.

\section{El Museo de los Niños de Costa Rica (MNCR) ${ }^{4}$}

El Museo de los Niños de Costa Rica es propiedad intelectual de la Fundación Ayúdanos para Ayudar. Cuenta con 11 años de funcionamiento. El objetivo general de esta institución es contribuir a la formación de seres humanos críticos, dinámicos, creativos y participativos, mediante la puesta en marcha de actividades complementarias a la educación formal (FAPA, 1993, pp. 30-31):

Para la cumplimiento de sus objetivos, el museo cuenta con todo el soporte administrativo y técnico profesional del Centro Costarricense de Ciencia y Cultura (CCCC), también de la Fundación Ayúdanos para Ayudar (FAPA). El CCCC cuenta con otros proyectos, ellos son: la Galería Nacional, Complejo Juvenil del Conocimiento y Jaula de la tecnología y el Auditorio Nacional.

Además, el museo se compone de las siguientes unidades y departamentos que diseñan y ejecutan actividades propias del museo en lo educativo:

1. Sección de museos: Encargada de la atención del público visitante mediante los "guías de museo".

2. Museografía y apoyo educativo: Se encarga del diseño, planificación y ejecución y curaduría de salas de exhibición. Posee su propio taller y diseñadores.

\footnotetext{
${ }^{4}$ Dirección Web: www.museocr.com
} 
3. Oficina de Actividades Culturales y Científicas (del CCCC): Se encarga de proyectos educativos específicos y piloto, de interés institucional.

Ahora bien, debido a que esta es la institución conocida por la autora, es la que se utiliza como punto de partida para la comparación con los museos y centros españoles. Para ellos se ofrece en el anexo I, una descripción detallada de aspectos museológicos relacionados con cuestiones educativas que servirán de punto de partida para el estudio de los otros museos.

\section{Museo de Ciencias Naturales en Barcelona (MCNB) ${ }^{5}$}

Es un museo que se abre con este nombre en el año 2000, producto de la unificación del Museo de Zoología y del Museo de Geología, ambos de 1878. Su presupuesto y lineamientos de trabajo, a diferencia de los otros museos y como una particularidad nacional, proviene del Ayuntamiento de Barcelona. Tiene como objetivo general preservar el patrimonio para dar a conocer sus colecciones zoológicas y geológicas, y dejar patente, de esta manera, su trascendencia para el desarrollo científico, económico y cultural de la sociedad.

Su exposición permanente es propia de la museología tradicional, pero sus temporales se diseñan, siguiendo lineamientos de la nueva museología.

Para su trabajo investigativo y educativo cuenta con varios equipos de trabajo: de colecciones, de la biblioteca, de publicaciones científicas, de investigación y de producciones culturales que ofrece tres programas: exposiciones, actividades educativas e imagen y comunicación. El énfasis de esta institución es la investigación, mientras que los programas educativos se diseñan e implementan mediante una subcontratación de servicios educativos de animación sociocultural.

\section{Museo Nacional de Ciencias Naturales en Madrid (MCNM) ${ }^{6}$}

El MNCN tiene como objetivo promover un conocimiento más completo de la diversidad del mundo natural para mejorar la percepción de la ciencia y de la riqueza del patrimonio natural. El Museo ha sufrido varias transformaciones, desde sus inicios en 1772 . Pertenece al Estado. Actualmente cuenta con tres vicedirecciones: investigación, colecciones

\footnotetext{
${ }^{5}$ Dirección Web:

http://bcnweb13.bcn.es:81/NASApp/wprmuseuciencies/Museu.GeneradorPagines?idioma=2
} 
y exposiciones y programas públicos. Su énfasis se encuentra en la investigación en ciencias naturales y suele subcontratar servicios educativos de animación socio-cultural y diseño museográfico.

Este museo y el anterior funcionan de manera similar en algunos aspectos, debido a que ambos son de una u otra forma estatales.

\section{Ciudad de las Artes y las Ciencias en Valencia (CACV) ${ }^{\mathbf{7}}$}

La CACV es un complejo cultural que además del Museo de las Ciencias Príncipe Felipe, ha diseñado un oceanográfico y tiene en proceso la construcción y diseño de otro proyecto; el Palacio de las Artes. Pertenece a la Generalitat Valenciana (el gobierno autónomo de Valencia).

El museo ha sido pensado como un espacio para que el visitante acceda al conocimiento científico de manera más divertida, para despertar su curiosidad y su interés, mediante su involucramiento en actividades que le permitan el aprendizaje.

Cada proyecto de la CACV cuenta con un departamento dedicado a las cuestiones educativas para el diseño de actividades y materiales didácticos y el museo dispone un departamento dedicado a la Museografía. Además, comprende un equipo asesor conformado por cinco premios Novel en el campo de las ciencias naturales.

\section{Parque de las Ciencias en Granada (PCG) ${ }^{8}$}

El Parque de las Ciencias fue inaugurado en 1995, con el interés de mostrar que la cultura científica puede ser algo ameno y divertido. Pertenece a un consorcio de empresas e instituciones, en las cuales la Junta de Andalucía aporta el 50\% del presupuesto, el Ayuntamiento el $25 \%$ y varios bancos el otro $25 \%$. El poder de decisión sobre el parque es proporcional al aporte de cada una de las empresas e instituciones. Ahora bien, el $48 \%$ aproximadamente de los egresos de la institución se logra autofinanciar.

Actualmente, se trabaja en un proyecto que se encuentra en fase de construcción arquitectónica y, que al finalizar, triplicará el tamaño actual. La estructura organizacional está también siendo redefinida, en función de los nuevos proyectos que se suman. Con respecto a los programas educativos, sí es claro que cuentan con un departamento de educación y otro de museografía.

${ }_{7}^{6}$ Dirección Web: http://www.mncn.csic.es/

7 Dirección Web: http://www.cac.es 


\section{Museo de Ciencia en Barcelona, Cosmocaixa (CB) ${ }^{9}$}

Este es el primer museo interactivo de España; tiene 25 años de fundado y pertenece a la Fundación privada "La Caixa". Uno de sus proyectos es el Cosmocaixa de Madrid, aunque funcionan con cierta independencia en términos de la propuesta educativa y la oferta museográfica.

Los objetivos del museo son estimular el conocimiento científico; conseguir la participación directa de ciudadanos y convertirse en un lugar de encuentro entre la ciencia y la sociedad.

Para aspectos educativos cuentan con un departamento de proyectos que se encarga de todo el quehacer museográfico y con otro departamento de didáctica que se responsabiliza de las actividades educativas complementarias a la museografía y del trabajo de los promotores culturales o guías del museo.

Un aspecto importante del CB y, muy particular, respecto a los museos que conoce la autora, se debe a que no cuenta con una exposición permanente, sino solo con exposiciones temporales. De esta manera, cada cierto tiempo hacen circular las exposiciones por España y diseñan nuevas.

\section{Museo de Ciencia de Madrid, Cosmocaixa (CM) ${ }^{10}$}

Como se mencionó, el $\mathrm{CM}$ es un proyecto que se deriva de $\mathrm{CB}$, con quienes establece lineamientos de trabajo.

Cuenta con un departamento de didáctica, encargado de los programas educativos. La museografía es diseñada en $\mathrm{CB}$, pero es diferente de la que se ofrece en Barcelona.

\section{Aporte de la nueva museología española al MNCR}

Con base en el sistema de comparación que se ofrece en el anexo II, a continuación se ofrece el análisis de aquellos aspectos que se considera pueden ser o no un aporte al quehacer museal del MNCR:

- Sobre políticas y objetivos: Si bien se indica que la mayoría de los museos cuentan con políticas y objetivos, realmente no están sistematizados claramente en un documento

\footnotetext{
${ }^{8}$ Dirección Web: http://www.servicios.nortecastilla.es/varios/museodelaciencia

${ }^{9}$ Dirección Web:

http://portal1.lacaixa.es/Docs/Chan/99/1-99-10-00000001.html?dest=1-99-10-00000000\#

${ }^{10}$ Dirección Web: http://www1.lacaixa.es:8090/webflc/wprOpres.nsf/wurl/mcal001 cat 
oficial que se pueda consultar. Salvo algunos esquemas generales de filosofía de trabajo que se pueden encontrar en CB (Moll y Codina, 2004; De Pablo, 2004; Wagensberg, 2004), PCG (Posse, Castillo y Paramo, 2004) y MNCR (Fundación Ayúdanos para Ayudar, 1993). Se ha encontrado que en España, las reflexiones pedagógicas y educativas en general del quehacer de la nueva museología, suelen realizarse desde la academia (Pérez, 2000; Pastor, 2000; Sabbatini, 2004) como pautas generales por seguir; pero no como una producción o reflexión autónoma de cada institución.

La importancia de contar con unas políticas y objetivos claros en el quehacer museal, constituyen el punto de partida para evaluar, mejorar y crecer especialmente en las formas de educar que puede ofrecer un museo.

- $\quad$ Sobre la estructura organizacional: Tal y como lo indica Pastor (2004, p.35), en la nueva museología lo normal es contar con departamentos dedicados a las cuestiones educativas que complementen con actividades didácticas a la propuesta museográfica de la institución. Por esta razón, es común la existencia no solo de encargados de museografía, sino también la presencia de responsables de otros aspectos educativos.

- $\quad$ Sobre la museografía se encontraron varios aspectos que pueden ser de interés para el MNCR, veamos ${ }^{11}$ :

a. El uso de videojuegos con clara intención educativa como un elemento ilustrativo en el contexto de una sala museográfica. Esta versión comprende más allá de un software de información animado o de un sistema de selección múltiple. Este recurso podría tener muy buena acogida por parte de los visitantes. El reto está en lograr diseños educativos adaptados a los tiempos de visita a la sala, ya que a diferencia de los que existen en el mercado para uso doméstico o escolar, deben tener una duración más corta.

b. Vitrinas cúbicas de comprensión súbita: Son vitrinas cúbicas que albergan una gran cantidad de objetos de un mismo tipo (por ejemplo: una colección de rocas organizada por color, forma, tamaño) dispuestos en forma de cuadrícula tridimensional. La idea de este juego, es que el visitante logre descifrar la lógica de ordenamiento de los objetos, según la información que se le ofrece y, de esa forma, pueda comprender "súbitamente" aspectos tales como por ejemplo el desarrollo tecnológico de instrumentos fabricados por el ser humano. Este es un 
recurso con un nivel de dificultad importante, por eso resultan ideales para trabajar con grupos de cuarto grado de primaria a secundaria. En este sentido, también el CB cuenta con "Vitrinas de comprensión súbita" que no son tridimensionales y que sería un recurso más adecuado para grupos de preescolar a tercer grado de educación primaria.

c. Exhibiciones itinerantes y temporales: La posibilidad de contar con estos tipos de exposiciones o no contar con ellas, además de una política clara al respecto, supone un costo muy elevado, para una institución como el MNCR. Sin embargo, esto no limita la posibilidad de que se puedan abrir espacios sistemáticos "extramuros" ${ }^{12}$ que permita llevar actividades y algunos recursos museográficos del MNCR a zonas alejadas de la capital.

d. Otro de los aspectos importantes a considerar por parte del MNCR es el recurso de establecer convenios con otros museos y centros de ciencia interactivos (i.e. el caso de la CAC que tiene convenio con el San Franklin Institute) para replicar exhibiciones vinculadas con avances científicos u otras temáticas de interés, efectuando las adaptaciones culturales respectivas. Esto ahorra costos en el plano del diseño museográfico y del trabajo en los guiones propios para la concreción de una exhibición (guiones científico, temático, museográfico y cedulario).

e. Estación metereológica, planetario y planetario burbuja: Estos recursos audiovisuales suponen una inversión millonaria que antes de conseguir fondos y llevarla a cabo, sería importante realizar un estudio de su impacto para valorarlo en el contexto de la planificación del MNCR. Sin embargo, el planetario burbuja es una propuesta educativa muy acertada para grupos de preescolar y primer grado de educación primaria, que no resulta tan costosa como las otras. Se considera como uno de los proyectos que el MNCR podría implementar, ya que la mayoría de grupos organizados que visitan el MNCR son de nivel preescolar y no existe un diseño museográfico sobre temas científicos especialmente para ellos.

f. Jardín de astronomía: EI PCG cuenta con un jardín de astronomía en su zona verde; resulta de gran interés para los niños y jóvenes que, en momentos de espera salen, visitan y juegan con los diferentes relojes y calendarios solares. La

\footnotetext{
${ }^{11}$ Interesa en la descripción, enfocar aquellos aspectos que aparecen negativos para el MNCR en le paradigma de comparación.

${ }^{12}$ Es un tema que ha sido tratado muchas veces, pero no se ha concretado un proyecto específico, únicamente en el área de divulgación y algunas experiencias piloto.
} 
idea de contar con una exhibición de juegos diseñados para ser ubicados al aire libre y a la vez, aprender sobre algún tema, es algo que podría ser de interés al MNCR, ya que es una de las solicitudes del público visitante "contar con espacios al aire libre para jugar". Aunque si bien existe el espacio para ponerlo en escena, no existe condiciones de seguridad pública para usarlo.

- Sobre las actividades educativas: Resulta importante retomar de la experiencia española, la riqueza de sus talleres científicos con experimentos variados y objetos originales para ser analizados por los estudiantes (por ejemplo fósiles de animales y huellas, etc.). Cabría retomar del acerbo biológico, geológico y cultural costarricense elementos que se vinculen con las salas y enriquecer la propuesta de talleres. Uno de los aspectos diferentes es el nivel educativo de los promotores, los museos españoles, por lo general, contratan profesionales; mientras que el MNCR contrata estudiantes. Sin embargo, en los museos españoles se nota la diferencia entre el grado académico de los profesionales en educación (tienden a ser licenciados) y los de investigación y museografía (tienden a ser doctores). Resulta de interés revisar, si el nivel educativo influirá en la calidad de la propuesta educativa.

Ahora bien, los talleres conservan la estructura tradicional; la previsita que es un documento guía que se entrega a los docentes para que realicen actividades antes de visitar el Museo y así la actividad en el Museo tiene un precedente que la enriquece. $Y$ las actividades posvisita recomendadas que suelen servir de conexión con la vida escolar y el taller. Se encontró que en algunos casos, se omite la previsita o la posvisita ya que muchos docentes no las ejecutan.

Otro de los aspectos importantes, en lo educativo, es el diseño museográfico de espacios, los cuales son dedicados únicamente a la implementación de talleres. Así, salas como el "¡Toca, Toca!" y el "Clic" son espacios donde los niños en grupos pequeños y con biólogos pueden tocar, oler, ver y escuchar animales vivos (serpientes, tortugas grandes, animales marinos, etc.) mientras estudian sus formas de vida. Se considera que para un taller estos espacios son realmente ricos porque permiten profundizar en los temas planteados mediante un mayor contacto y relación con una colección viva y para explotar recursos que no pueden exponerse al público en general por su particular delicadeza. 
- Sobre las publicaciones y documentos: Como se indicó en el punto primero, no existe documentación académica y teórica sobre el quehacer educativo y museológico de producción autónoma. Sin embargo, lo que sí fue posible encontrar, fueron materiales didácticos muy diversos y con alta calidad no solo en contenidos, sino también en diseño e impresión. Un tipo de publicación que pareciera ser muy propia de la nueva museología española es la "Guía didáctica del museo" con un fuerte objetivo de divulgación científica. Este documento le ofrece al lector información científica sobre los temas tratados en cada una de las salas y, en la mayoría de los casos, actividades o experimentos para realizar en casa o en la escuela sobre cada tema.

Por otro lado, resulta interesante que en cuatro museos españoles muchos de los guiones científicos ${ }^{13}$ de las salas, suelen publicarse como artículos de divulgación. Esto es algo que se puede hacer en el MNCR, promover publicaciones, desde los estudios que supone el trabajo museográfico.

- Formas de financiamiento: el sistema de becar estudiantes avanzados (con nivel de bachillerato universitario) por períodos lectivos, resulta atractivo, no solo en términos económicos, sino como una forma de contar con un porcentaje de guías de sala que puedan dedicarse a talleres y demostraciones de pasillo especializadas. Otro formato de financiamiento de personal es el contrato para prácticas de formación que se utiliza en el MCNB, y que financia el Ayuntamiento mediante el programa de formación ocupacional teórico-práctico que implementa "Barcelona Activa" y que facilita personal en formación con un salario. De igual manera, en el MNCR se podría lograr establecer un convenio para prácticas profesionales de formación con el Instituto Nacional de Aprendizaje de Costa Rica, que permita el adiestramiento de estudiantes en algunos de sus programas afines según los intereses de ambas partes.

\footnotetext{
${ }^{13}$ En un diseño museográfico el guión científico es la investigación base del tema por tratar en una sala.
} 


\section{Conclusiones}

En términos generales, la propuesta educativa y museográfica entre los museos involucrados en el estudio, es muy similar; a diferencia del CB, que se ha esforzado por plantear su propio estilo museográfico. Algunas diferencias de la oferta educativa están vinculadas con las posibilidades presupuestarias que en el caso de CB, CM, PCG y CACV es superior y no es motivo de preocupación cotidiana.

Así, con base en el aporte de la nueva museología española al MNCR, se puede notar la necesidad de promover una cultura científica al interno de las instituciones involucradas. Lo anterior se hace notorio, al no encontrarse publicaciones y documentación desde los museos que muestren y den fe de sus bases pedagógicas y filosóficas, de lineamientos de diseño museográfico y de la acción museológica en general. Se asume como verdad lo que se piensa y como correcto lo que se hace, salvo "ciertos detalles por mejorar" como se afirma.

El proceso de mejoramiento de la propuesta educativa pareciera muy intuitivo y poco sistemático, ya que se usan criterios tales como "lo que pega" y "lo que gusta" en lo museográfico y en las actividades "como se sintió" y si "gustó". En este sentido, la excepción sería que se suelen pasar cuestionarios a los docentes para evaluar los talleres. Pero no existen programas de evaluación sistemáticos que sustenten la toma de decisiones.

Con respecto a la museografía, aún no se ve el avance hacia diseños que faciliten las interacciones de grupos de personas, tal y como se está planteando en otros ámbitos (Borum, M. y otros, 1998). Se mantiene el diseño para uso individual y una fuerte orientación hacia público escolar (primaria y secundaria), dejando marginados a los grupos familiares y personas con alguna limitación. Lo anterior resulta contradictorio considerando el hecho de que todos los museos coinciden en que es su mayor población visitante son familias. Sobre poblaciones con limitaciones solo se retoma la disposición de rampas de acceso y ascensores especiales para sillas de ruedas en museos europeos.

Ahora bien, existen muchos aportes que pueden enriquecer la propuesta educativa del MNCR. En el ámbito museográfico; las vitrinas de comprensión súbita, los videojuegos, áreas de exposición para niños de edad preescolar y áreas de juego externas pensadas como espacios museográficos.

En lo que respecta a las actividades educativas, existe mucha experiencia en el diseño de experimentos de ciencias naturales, debido al nivel educativo de sus diseñadores, a sus profesiones y a las colecciones museográficas que se utilizan para efectuarlos. Y, por último, el modelo de diseño de las publicaciones que sirve de complemento a los talleres, el 
planetario y las salas, proporciona ideas sobre el ordenamiento de la información y su vinculación con actividades prácticas, según la edad del público, en función de los intereses temáticos del museo.

Por último, es importante destacar que el uso de un paradigma de comparación permitió no solo una forma ordenada de observar, sino profundizar para poder identificar nuevos elementos y también ubicar en los aspectos similares diferencias particulares entre las instituciones. El propósito era lograr una sistematización de lo observado para identificar aportes concretos de la nueva museología española al MNCR y, en este sentido, el paradigma comparativo fue de mucha utilidad.

\section{Referencias}

Ariel, P. (2005). Nueva Museología, hacia un nuevo paradigma. Revista Digital Nueva Museología. Argentina. Recuperado el 1 de octubre de 2005, de http://www.nuevamuseologia.com.ar/nuevo paradigma.htm

Borum M., Dritsas J., Johnson J., Peter N., Wagner K., Fadigan K., Jangaard A., Stroup E. y Wenger A. (1998). Family Learning in Museums; The PISEC Perspective. Philadelphia-Camden Informal Science Education Collaborative (PISEC). The Franklin Institute. Estados Unidos de América.

De Pablo, P. (2004). Qué ciencia enseñar y cómo hacerlo. Cuadernos de pedagogía. No.340, noviembre. España: CISSPRAXIS, S.A. Pp. 64-67.

Falk, J. y Dierking, L. (2000). Learning From Museums: Visitors Experiences and the Making of Meaning. Estados Unidos: Altamira Press.

Fundación Ayúdanos para Ayudar. (1993). Proyecto Centro Costarricense de Ciencia y Cultura. Documento interno e inédito. Costa Rica.

Moll, B. y Codina, B. (2004). Museos de ciencia y nuevos retos. Cuadernos de pedagogía. No. 340, noviembre. España: CISSPRAXIS, S.A., pp. 54-55.

Pastor, M. (2004). Pedagogía museística: nuevas perspectivas. España: Editorial Ariel S.A.

Pérez, E. (2000). Estudios de visitantes en museos: metodología y aplicaciones. España: Ediciones Trea.

Posse, P. Castillo, D. y Páramo, E. (2004). El método como curiosidad. En: Cuadernos de Pedagogía. No. 340, noviembre. España: CISSPRAXIS, S.A. Pp. 60-63.

Rodríguez, K. (2000). Programa de visitas autoguiadas para el Museo de los Niños. Proyecto de Graduación para optar por el grado de Licenciada en Ciencias de la Educación con Énfasis en Administración de Programas de Educación no Formal. Costa Rica: Universidad de Costa Rica. 
Sabbatini, M. (2003). Centros de ciencia y museos científicos virtuales: teoría y práctica. Revista Teoría de la Educación: educación y cultura en la sociedad de la información. Universidad de Salamanca. (4). Recuperado el 1 de marzo de 2004, de http://www3.usal.es/ teoriaeducacion/rev numero 04/n4 art sabbatini.htm

Sabbatini, M. (2004). Museos y centros de ciencia virtuales. Complementación y potenciación del aprendizaje de ciencias a través de experimentos virtuales. Tesis presentada para optar por el grado de doctor. Universidad de Salamanca. España: Instituto Universitario de Ciencias de la Educación.

Spradley, J. (1980). Participant Observation. New York, Estados Unidos: Holt, Rinehart and Winston.

Wagensberg, J. (2004). Esa herramienta de cambio. Cuadernos de pedagogía. No. 340, noviembre. España: CISSPRAXIS, S.A. Pp 56-59. 


\section{Anexo I \\ Guía de observación etnográfica con base en el Museo de los Niños de Costa Rica}

1. Políticas y objetivos institucionales: Se refiere a los lineamientos y fines educativos que orientan el diseño y la ejecución de los programas y proyectos educativos del Museo propiamente (exposición y actividades complementarias).

2. Estructura organizacional solo de programas y proyectos educativos:

a. Unidad o departamento de museografía y taller de diseño y reparación.

b. Unidad o departamento encargado de programas y proyectos educativos complementarios a la museografía

c. Unidad o departamento dedicada a la atención del público en su visita al museo

3. Museografía: Se refiere al diseño, construcción y montaje de las exhibiciones. La clasificación del tipo de exhibiciones que se ofrece categoriza tipos de exposición, según los aspectos dominantes y, a la vez, subcategoriza según los posibles aspectos diferenciadores de cada una. Ahora bien, es posible encontrar en una sala, elementos de los tres tipos que se proponen. Sin embargo, para efectos clasificatorios y para simplificarle al lector la comprensión, la categorización se plantea de acuerdo con las tendencias generales que se pueden encontrar en las museografías:

a. Exhibiciones interactivas contextuales: Son salas cuyo eje central es recrear escenarios de la vida cotidiana y mostrar, a los educandos, los objetos museográficos en contexto "real", en el cual se puede jugar y participar de él. Con este fin, pueden recurrir a formatos museales indicados en los puntos siguientes. Por ejemplo, la recreación de un consultorio de dentista o de una casa indígena. Se pueden utilizar diferentes elementos que faciliten la comprensión de la escena: disfraces, juguetes de la época, entre otros.

b. Exhibiciones interactivas sobre temas diversos: Son exhibiciones que procuran desarrollar contenidos sobre un tema y, para ello, recurren a Volumen 5, Número 2, Año 2005, ISSN 1409-4703 22 
diversos recursos que faciliten oportunidades de interacción. Los recursos por utilizar serían:

i. Experimento: Es una actividad presencial y participativa que permite plantearse hipótesis sobre el fenómeno observado. Se muestran o simulan conceptos, procesos o principios científicos o tecnológicos. Por ejemplo: excavar y encontrarse restos fósiles, juegos de electromagnetismo, entre otros.

ii. Dispositivos electrónicos: Son los objetos que, mediante la activación de elementos electrónicos o mecánicos, activan sonido o imágenes. Así, permiten observar un diorama o encontrar una respuesta a una pregunta.

iii. Dispositivos de simulación virtual: Son operados mediante software. Por ejemplo, un juego educativo para ser operado por computadora.

iv. Replicas de objetos: Son objetos museográficos que replican uno original, puesto que, por su valor patrimonial, unicidad no es posible contar con el para que los educandos puedan explorarlo con la mayoría de los sentidos. Por ejemplo: una vasija precolombina o la máscara de Tutankamón.

v. Objetos originales de exploración. Tales como: un motor, un tren, etc.

vi. Modelos de elementos o sistemas microscópicos o macroscópicos. Por ejemplo: un modelo del sistema solar y una molécula de agua.

vii. Dispositivos para manipular manualmente. Por ejemplo: rompecabezas bi o tridimensionales; un gavetero con información sobre un tema; puertas con preguntas y respuestas, etc.

viii. Dispositivos de simulación presencial: Son operados mediante software o simulación analógica. Por ejemplo: robot que simula ser un personaje conocido o lluvia en un "bosque".

c. Exhibiciones tradicionales de observación y escucha: Son aquellas exhibiciones de la museología tradicional que cuentan con objetos o aspectos que únicamente se emplean para ser observados y, en algunos casos, escuchados. Utiliza recursos tales como; murales, meteorito en vitrina, audiovisuales, entre otros. 
i. Objetos originales: Para observarlos por su valor, no pueden ser manipulados. Por ejemplo: un traje espacial.

ii. Recursos audiovisuales.

d. Exhibiciones permanentes: Es la propuesta museográfica de planta que ha sido diseñada para que se mantenga en el tiempo y que suele no variar en su concepto original sino mejorarse o mantenerla.

e. Temáticas de todas las exhibiciones están vinculadas con el currículo oficial del sistema educativo formal para primaria.

4. Actividades educativas: Se trata de todas las actividades planificadas con intenciones educativas que complementan la museografía, al interés del público o contribuyen al cumplimiento de los objetivos educativos del museo. Existen varias modalidades:

a. Para todo público (masivas): Al igual que, en la museografía, es posible que una de las categorías de este punto contenga en su implementación varias de las otras. Por ejemplo, un festival puede tener talleres y conciertos sobre el tema a tratado.

i. Festivales: Son celebraciones que incluyen actividades principalmente lúdicas, orientadas hacia un objetivo educativo y con un tema central. Por ejemplo: Festival de la Tierra y Festival de Dinosaurios.

ii. Ferias: Son actividades con el fin de facilitar el acceso del público al conocimiento de la oferta y, eventualmente, compra, de bienes de importancia cultural-educativa. Por ejemplo, la Feria del libro.

iii. Talleres: Son programas que incluyen aspectos teóricos que suelen tratarse en las salas de exhibición, sobre un tema y una aplicación práctica de ellos. Por ejemplo: "Tradiciones Navideñas", "Vistámonos de indígenas", "Caricaturas", etc.

iv. Conciertos: Son demostraciones musicales del repertorio de la sociedad local y de la cultura universal.

v. Visitas guiadas: Son recorridos por el Museo en grupos con un guía. La visita guiada es general por todo el Museo.

vi. Guía libre: Se refiere a la visita libre del público (ellos seleccionan cual sala desean visitar) y a la presencia de guías en la sala para la atención de quiénes deseen ingresar. 
vii. Demostraciones de pasillo: Son breves actividades de teatro, de títeres, experimentos, entre otras, las cuales se realizan para el complemento de alguno de los temas expuestos en el museo. La idea central es sorprender al público, mediante una presentación caracterizada por intentar despertar en los educandos, el juego, la diversión, la curiosidad y la pregunta.

b. Actividades educativas para niños

i. Talleres: Ver punto 3.c Además, es importante realizar esta modalidad didáctica referida a cualquier aspecto que se pueda ubicar entre los objetivos centrales de la institución. De esta manera, aunque no hay una sala de ajedrez, se puede ofrecer un taller de ello, en tanto que el interés es contribuir a la formación integral de los niños y niñas.

ii. Certamen de pintura: Es un concurso de pintura, en donde se define la técnica por utilizar, el tema o si es libre, y se participa por grupos de edad. Se cuenta con un jurado y una premiación.

iii. Certamen literario: Es un concurso de creación literaria, en donde se define el género literario, el tema o si es libre, y se participa por grupos de edad. Se cuenta con un jurado y una premiación.

iv. Programas vacacionales: Son programas con fuerte contenido lúdicoeducativo, para organizar las vacaciones de los niños y las niñas. (campamentos, giras educativas, etc.).

c. Actividades educativas para grupos escolares

i. Talleres que complementan los programas del sistema educativo oficial. Ver punto 2.C.

ii. Visitas guiadas. Ver punto 2.e.

iii. Visita temática que supone un recorrido que utiliza un tema como eje central. Por ejemplo: "Cuerpo humano" (se visita la sala sobre ese tema, otra sobre salud buco dental y otra sobre gestación).

iv. Guía libre. Ver punto $2 . f$

v. Actividades en las escuelas 
d. Publicaciones (Rodríguez, 2000) y documentos:

i. Ayudas didácticas para maestros.

ii. Material didáctico para escolares.

iii. Para padres y madres, sobre temas de crianza y educación de los hijos en el museo.

iv. Divulgación y comunicación de la institución al público en general o grupos específicos (oferta educativa).

v. Guías didácticas para salas de exhibición dirigidas al público en general.

vi. Folletos didácticos para programas educativos específicos.

5. Formas de financiamiento

a. Financiamiento estatal.

i. Partidas específicas para el desarrollo de proyectos

ii. Partidas específicas para los gastos administrativos

iii. Personal

b. Financiamiento privado

i. Donaciones: Dádiva de una persona o institución al Museo para la realización de uno o cualquier proyecto. No se espera nada a cambio.

1. En especie

2. En efectivo

ii. Patrocinio: Apoyo económico y de ejecución por parte de empresas o personas para la realización de un proyecto afín a sus valores o intereses. Se espera una retribución a cambio del patrocinio. Por ejemplo: presencia de marca, uso de instalaciones, etc. Las "alianzas estratégicas" entran en esta categoría.

c. Voluntariado

1. Mediante convenios interinstitucionales. I.E. con universidades.

2. Libre: Personas que deciden apoyar al museo, mediante la realización de su trabajo, sin esperar retribución económica.

d. Autofinanciamiento

i. Taquilla: El ingreso obtenido por la venta de las entradas al museo.

ii. Venta de servicios: Implementación de programas educativos y organización de eventos. 
iii. Alquiler de espacios para eventos privados.

iv. Concesión de espacios para tienda y cafetería.

6. Investigación y evaluación de programas educativos (diagnóstico, proceso, producto).

a. Evaluación.

i. De los objetivos de los programas y proyectos.

ii. Sobre la implementación de los programas y proyectos.

1. Por departamento o unidad.

2. Institucional externa.

3. Institucional interna.

b. Investigación.

i. Iniciativa institucional.

1. Estudios de mercado.

2. Estudios para diseño de salas.

3. Estudios de conocimiento del público.

ii. Iniciativa departamental.

1. Estudios de mercado.

2. Estudios de conocimiento del público.

iii. Iniciativa individual (tesis de grado) con apoyo institucional. 


\section{ANEXO II}

Museos y centros interactivos españoles: aportes al Museo de los Niños C.R.

Paradigma de comparación

\begin{tabular}{|c|c|c|c|c|c|c|c|}
\hline $\begin{array}{c}\text { Museo } \\
\text { Característica }\end{array}$ & $\begin{array}{c}1 \\
\text { MNCR }\end{array}$ & $\begin{array}{c}2 \\
\text { MCNB }\end{array}$ & $\begin{array}{c}3 \\
\text { MCNM }\end{array}$ & $\begin{array}{l}4 \\
\text { CACV }\end{array}$ & $\begin{array}{c}5 \\
\text { PCG }\end{array}$ & $\begin{array}{c}6 \\
\text { CM }\end{array}$ & $\begin{array}{c}7 \\
\text { CB }\end{array}$ \\
\hline 1. Políticas y objetivos & + & + & + & + & + & - & + \\
\hline 2. Estructura organizacional & + & + & + & + & + & $\begin{array}{c}+/- \\
14\end{array}$ & + \\
\hline a. Museografía y taller & + & + & + & + & + & - & + \\
\hline b. Educación complementaria & + & + & + & + & + & + & + \\
\hline $\begin{array}{l}\text { c. Atención a visitantes en el } \\
\text { museo }\end{array}$ & + & - & + & + & + & + & + \\
\hline 3. Museografía & + & + & + & + & + & + & + \\
\hline $\begin{array}{l}\text { a. Exhibiciones interactivas } \\
\text { contextuales }\end{array}$ & + & + & + & + & + & + & + \\
\hline $\begin{array}{l}\text { b. Exhibiciones interactivas } \\
\text { temáticas }\end{array}$ & + & + & + & + & + & + & + \\
\hline i. Experimentos & + & - & - & + & + & + & + \\
\hline $\begin{array}{r}\text { ii. } \begin{array}{r}\text { Dispositivos } \\
\text { electrónicos }\end{array} \\
\end{array}$ & + & + & + & + & + & + & + \\
\hline iii. Simulación virtual & + & + & + & + & + & + & + \\
\hline iv. Réplicas & + & + & + & + & + & + & + \\
\hline v. Objetos originales & + & + & + & + & + & + & + \\
\hline vi. Modelos & + & - & - & + & + & + & + \\
\hline $\begin{array}{l}\text { vii. Dispositivos } \\
\text { manipulación }\end{array}$ & + & - & + & + & + & + & + \\
\hline viii. Simulación presencial & + & + & + & + & + & + & + \\
\hline $\begin{array}{ll}\text { ix. } & \text { Videojuegos } \\
& \text { educativos }\end{array}$ & - & + & + & - & + & + & - \\
\hline x. Vitrinas cúbicas de & - & - & - & - & - & - & + \\
\hline
\end{tabular}

14 Si bien cuentan con una estructura de organización del trabajo, no se ha concretado en un organigrama oficial. 


\begin{tabular}{|c|c|c|c|c|c|c|c|}
\hline comprensión súbita & & & & & & & \\
\hline c. Exhibiciones permanentes & + & + & + & + & + & + & - \\
\hline d. Exhibiciones tradicionales & + & + & + & + & - & + & + \\
\hline i. Elementos originales & + & + & + & + & + & + & + \\
\hline $\begin{array}{ll}\text { ii. } & \text { Recursos } \\
& \text { audiovisuales }\end{array}$ & + & + & + & + & + & + & + \\
\hline $\begin{array}{c}\text { e. Temática vinculada con } \\
\text { educación formal }\end{array}$ & + & + & + & + & + & + & + \\
\hline f. Exhibiciones itinerantes & - & + & + & + & - & - & + \\
\hline g. Exhibiciones temporales & - & + & + & + & + & + & + \\
\hline h. Planetario & - & - & - & + & + & + & + \\
\hline i. Planetario burbuja & - & - & - & - & - & + & + \\
\hline j. Jardín de la astronomía & - & - & - & - & + & - & - \\
\hline $\begin{array}{l}\text { k. Estación metereológica/ } \\
\text { astronómica }\end{array}$ & - & - & - & + & + & + & - \\
\hline 4. Actividades educativas & + & + & + & + & + & + & + \\
\hline a. Masivas & + & + & + & + & + & + & + \\
\hline i. Festivales & + & + & + & + & $+/-15$ & + & - \\
\hline ii. Ferias & + & + & + & + & - & + & - \\
\hline iii. Talleres & + & + & + & + & + & + & + \\
\hline iv. Conciertos & + & - & + & + & + & + & - \\
\hline v. Visitas guiadas & + & + & - & + & - & + & + \\
\hline vi. Guía libre & + & + & + & + & + & + & + \\
\hline $\begin{array}{l}\text { vii. Demostraciones de } \\
\text { pasillo }\end{array}$ & + & + & - & - & - & - & + \\
\hline $\begin{array}{l}\text { viii. Tertulias, } \\
\text { conferencias, cursos }\end{array}$ & - & + & + & + & + & + & + \\
\hline $\begin{array}{l}\text { ix. Programas para } \\
\text { Cassals }\end{array}$ & 0 & + & + & + & + & + & + \\
\hline 5. Actividades educativas para niños & + & + & + & + & + & + & + \\
\hline a. Talleres & + & + & + & + & + & + & + \\
\hline
\end{tabular}

15 Realizan una actividad similar a un festival, el día que celebran el aniversario de apertura del parque. Ese día, se invita a docentes para realizar talleres sobre ciencias para otros docentes. 


\begin{tabular}{|c|c|c|c|c|c|c|c|}
\hline b. Certámenes de pintura & + & - & - & - & + & - & - \\
\hline c. Certámenes literarios & + & + & - & - & - & - & + \\
\hline d. Programas vacacionales & + & - & + & - & - & + & + \\
\hline e. Consejo infantil & $+/-^{16}$ & - & - & - & + & - & - \\
\hline $\begin{array}{l}\text { 6. Actividades educativas para grupos } \\
\text { escolares }\end{array}$ & + & + & + & + & - & + & + \\
\hline a. Talleres & + & + & + & + & + & + & + \\
\hline b. Visitas guiadas & + & + & _ & + & - & - & + \\
\hline c. Visitas temáticas & + & + & + & + & + & - & + \\
\hline d. Guía libre & + & + & + & + & + & + & + \\
\hline e. Actividades en escuelas & + & - & - & - & - & - & - \\
\hline f. Museografía para talleres & - & - & + & + & + & + & + \\
\hline 7. Publicaciones y documentos & + & + & + & + & + & + & + \\
\hline a. Ayudas didácticas a maestros & + & - & + & + & + & + & + \\
\hline $\begin{array}{l}\text { b. Material didáctico para } \\
\text { escolares }\end{array}$ & + & + & + & - & + & + & + \\
\hline $\begin{array}{l}\text { c. Divulgación y comunicación } \\
\text { externa }\end{array}$ & + & + & + & + & + & + & + \\
\hline $\begin{array}{l}\text { d. Guías didácticas para salas de } \\
\text { exhibición }\end{array}$ & + & - & + & + & & + & + \\
\hline $\begin{array}{l}\text { e. Folletos didácticos para } \\
\text { programas específicos }\end{array}$ & + & + & + & + & + & + & + \\
\hline f. Para padres y madres & - & + & + & - & - & - & - \\
\hline g. Sobre museología & - & - & - & - & - & - & + \\
\hline $\begin{array}{l}\text { h. Divulgación científica de } \\
\text { investigaciones de colecciones }\end{array}$ & - & + & + & + & - & - & + \\
\hline i. Guía didáctica del museo & - & - & + & + & + & + & $+/-^{17}$ \\
\hline 8. Biblioteca & + & + & + & + & + & - & $+/-^{18}$ \\
\hline 9. Financiamiento & + & + & + & + & + & + & + \\
\hline a. Estatal & + & + & + & + & + & - & - \\
\hline
\end{tabular}

${ }^{16}$ Existe un proyecto escrito que no se ha ejecutado.

${ }^{17}$ Está en proceso de diseño y publicación.

${ }^{18}$ Disponible solo para los funcionarios de la institución. 


\begin{tabular}{|c|c|c|c|c|c|c|c|}
\hline $\begin{array}{l}\text { i. Partidas específicas } \\
\text { para proyectos }\end{array}$ & + & + & + & + & - & - & - \\
\hline ii. Gastos administrativos & + & + & + & + & + & - & - \\
\hline iii. Personal & + & + & + & + & + & - & - \\
\hline b. Financiamiento privado & + & - & + & + & + & + & + \\
\hline i. Donaciones & + & - & + & + & - & - & + \\
\hline 1. Especie & + & - & + & + & - & - & + \\
\hline 2. Efectivo & + & - & + & + & - & - & + \\
\hline ii. Patrocinio & + & - & + & + & + & + & + \\
\hline c. Voluntariado & + & - & + & $+/-$ & + & - & + \\
\hline $\begin{array}{l}\text { i. Convenios } \\
\text { interinstitucionales }\end{array}$ & + & + & + & + & + & - & + \\
\hline ii. Libre & + & - & + & - & + & - & - \\
\hline iii. Adultos mayores & - & - & - & - & - & - & + \\
\hline d. Practicas profesionales & + & + & + & + & + & + & - \\
\hline e. Becarios & - & - & - & - & + & - & - \\
\hline $\begin{array}{l}\text { f. Contratos para prácticas de } \\
\text { formación }\end{array}$ & - & + & - & - & - & - & + \\
\hline g. Autofinanciamiento & + & + & + & + & + & + & + \\
\hline i. Taquilla & + & + & + & + & + & + & + \\
\hline ii. Venta servicios & + & - & - & + & - & - & - \\
\hline iii. Alquiler de espacios & + & - & + & + & - & - & - \\
\hline $\begin{array}{l}\text { iv. Concesión tienda- } \\
\text { cafetería }\end{array}$ & + & + & + & + & + & + & + \\
\hline $\begin{array}{l}\text { v. Venta servicios } \\
\text { técnicos }\end{array}$ & - & + & + & + & - & - & - \\
\hline 9. Investigación y evaluación & + & + & + & + & + & + & + \\
\hline a. Evaluación & + & + & - & + & + & + & + \\
\hline i. Objetivos & + & + & - & + & + & + & + \\
\hline ii. Implementación & + & + & - & + & + & + & + \\
\hline $\begin{array}{l}\text { 1. Por } \\
\text { departamento o } \\
\text { unidad }\end{array}$ & + & + & - & + & + & + & + \\
\hline
\end{tabular}




\begin{tabular}{|c|c|c|c|c|c|c|c|}
\hline $\begin{array}{l}\text { 2. Institucional } \\
\text { externa }\end{array}$ & + & + & - & - & + & + & - \\
\hline $\begin{array}{l}\text { 3. Institucional } \\
\text { interna }\end{array}$ & + & + & - & + & + & + & + \\
\hline b. Investigación & + & + & - & + & + & - & + \\
\hline i. Iniciativa institucional & + & + & - & + & + & - & - \\
\hline $\begin{array}{l}\text { 1. Estudios de } \\
\text { mercado }\end{array}$ & + & - & - & + & - & - & - \\
\hline $\begin{array}{l}\text { 2. Estudios para } \\
\text { diseño de salas }\end{array}$ & + & + & + & + & + & - & + \\
\hline $\begin{array}{l}\text { 3. Estudios de } \\
\text { público }\end{array}$ & + & + & - & + & - & - & + \\
\hline $\begin{array}{l}\text { i. Estudios de } \\
\text { mercado }\end{array}$ & + & - & - & + & - & - & - \\
\hline $\begin{array}{l}\text { 2. Investigación } \\
\text { educativa }\end{array}$ & + & - & - & - & - & - & - \\
\hline $\begin{array}{l}\text { 3. Estudios de } \\
\text { público }\end{array}$ & + & + & + & + & + & - & + \\
\hline iii. Iniciativa individual & + & - & + & - & - & - & + \\
\hline
\end{tabular}

Códigos utilizados: "-"ausencia de rasgo; "+" presencia de rasgo; "0" no aplica el rasgo por las características de la institución; "+/-“presencia del rasgo bajo ciertas restricciones o condiciones, las cuales se explican en el pié de página. La numeración de los museos se corresponde de la siguiente manera:

1. Museo de los Niños (Costa Rica).

2. Museo de Ciencias Naturales (Barcelona).

3. Museo Nacional de Ciencias Naturales (Madrid).

4. Ciudad de las Artes y las Ciencias (Valencia)

5. Parque de las Ciencias (Granada)

6. Museo de Ciencia (Cosmocaixa Madrid)

7. Museo de Ciencia (Cosmocaixa Barcelona) 